\title{
On the Whitney-Schwartz Theorem
}

By

\author{
Takao KAKITA*
}

Let $F$ be a closed set in $R^{n}$. Then, according to L. Schwartz [6], $F$ is called regular if for each $x \in F$ there are numbers $d(>0), \omega(\geqq 0)$ and $q(\geqq 1)$ such that any two points $y, z$ of $F$ with $r_{x y} \leqq d$ and $r_{x z} \leqq d$, are joined by a rectifiable curve in $F$, of length not greater than $\omega r_{y z}{ }^{1 / q}$ where $r_{x y}$ is the distance between $x$ and $y$. This definition is a generalization of "Property (P) local" by $\mathrm{H}$. Whitney ([9]). Schwartz stated in $[6]$ the following theorem without proof.

Theorem (Whitney-Schwartz). Let $T$ be a distribution in $R^{n}$ of order $m$ whose support is contained in a compact regular set $F$. Then

(A) $\left\langle T, \varphi_{\jmath}\right\rangle \rightarrow 0$ provided $\varphi_{\jmath} \in C^{\infty}\left(R^{n}\right)$ and their derivatives of order not greater than $m^{\prime}$ converge to zero uniformly on $F$, where $m^{\prime}$ is any integer $\geqq q(F) m$ and $q(F)$ is a number $\geqq 1$, depending on $F$.

(B) $T$ is represented by a finite sum of derivatives of measures whose supports are contained in $F$.

A similar result to the part (A) of Theorem was given for a general compact set $F$ by G. Glaeser, in such a sense that it has an advantage not making the behavior of $\varphi$, interfered in a 'neighborhood' of $F$ (see Proposition II, Chap III in $[2]$ ). We shall give an elementary proof of Theorem for a distribution in an open set $\Omega$ of $R^{n}$. For the proof we make use the reproduction of Whiteny's extension theorem by L. Hörmander $([4])$. The key lemma is the following:

Lemma. Let $u$ be a distribution of order $m$ in $\Omega$ with support in a compact regular set $F \subset \Omega$. Then there is a constant $C$ depending on $m^{\prime}$ and $F$ such that for any $\varphi \in C^{\infty}(\Omega)$

$$
|\langle u, \varphi\rangle| \leqq C\|\varphi\|_{m^{\prime}, F}
$$

where $q=q(F)$ is a positive number depending on $F, m^{\prime}$ is any integer $\geqq q m$, and

$$
\|\varphi\|_{k, F}=\sum_{|n| \leqq k} \sup _{x \in F}\left|(\partial / \partial x)^{\alpha} \varphi(x)\right| .
$$

Communicated by S. Matsuura, November 10, 1989, Revised April 8, 1991. 1991 Mathematics Subject Classification : 46F05.

* Department of Information and Computer Sciences, School of Science and Engineering, Waseda University, Shinjuku-ku, Tokyo, 160, Japan. 
In case $F$ is a closed ball, a simple proof of (1) was given by S. Mizohata in proving that evolution equations with finite propagation speed should be of kowalevskian type (see [5]). Now we shall restate the Whitney-Schwartz theorem in our form.

Theorem. Let $u$ be a distribution in $\Omega$ of order $m$ with support in a compact regular set $F \subset \Omega$. Then

(A) $\left\langle u, \varphi_{j}\right\rangle \rightarrow 0$ provided $\varphi_{j} \in C^{\infty}(\Omega)$ and their derivatives of order not greater than $m^{\prime}$ converge to zero uniformly on $F$, where $m^{\prime}$ is any integer $\geqq q(F) m$.

(B) $u$ is represented by a finite sum of derivatives of measures in $\Omega$ whose supports are in $F$.

Proof of (A) is immediate from Lemma. For proving (B) we can apply the Hahn-Banach theorem to the inequality (1) through the well-known method. We omit the details (see [6]).

Before proceeding to prove our lemma, we shall give a sketch of the partition of unity by Whitney in [8], following the reproduction by Hörmander.

Let $A$ be a closed set in $R^{n}$. The partition of unity is constructed as follows. First, divide $R^{n}$ into $n$-cubes of side 1 , and let $K_{0}$ be the set of all those cubes whose distance from $A$ are at least $\sqrt{n}$. Next, divide the remaining cubes into $2^{n}$ cubes of side $1 / 2$, and let $K_{1}$ be the set of those distant from $A$ at least $\sqrt{n} / 2$. Repeating such a division process, we have a series of the sets $\left\{K_{0}, K_{1}, \cdots\right\}$ where the union of all cubes of them is $R^{n} \backslash A$. Arrange all cubes in order of a series $Q_{1}, Q_{2}, \cdots$; the center and side of each $Q_{3}$ are denoted by $y^{j}$ and $s_{\jmath}$, respectively. Now take $\chi_{0} \in C_{0}^{\infty}$ being equal to 1 on the cube

$$
\left|x_{i}\right| \leqq 1 / 2, \quad i=1, \cdots, n
$$

and vanishing outside the cube

$$
\left|x_{\imath}\right| \leqq 1 / 2+1 / 8, \quad i=1, \cdots, n .
$$

Then define $\chi_{0} \in C_{0}^{\infty}\left(R^{n}\right)$ by

$$
\chi_{\jmath}(x)=\chi_{0}\left(\frac{x-y^{j}}{s_{\jmath}}\right) / \sum_{k=1}^{\infty} \chi_{0}\left(\frac{x-y^{k}}{s_{k}}\right), \quad j=1,2, \cdots .
$$

As for the denominator, it is verified

$$
1 \leqq \sum_{i=1}^{\infty} \chi_{0}\left(\frac{x-y^{k}}{s_{k}}\right) \leqq 4^{n} .
$$

The sequence $\chi_{j}$ in $C_{0}^{\infty}\left(R^{n}\right)$ is locally finite in $R^{n} \backslash A$ and has the properties :

(i) $\chi_{j} \geqq 0 ; \sum_{j=1}^{\infty} \chi_{j}(x)=1$ for $x \in R^{n} \backslash A$

(ii) for each $\alpha$, there is a constant $C_{\alpha}$ such that

$$
\sum_{j=1}^{\infty}\left|D^{a} \chi_{\jmath}(x)\right| \leqq C_{\alpha}\left(d(x, A)^{-|\alpha|}+1\right)
$$


for $x \in R^{n} \backslash A$ where $D=\partial / \partial x$

(iii) (the diameter of $\left.\operatorname{supp} \chi_{j}\right) \leqq C d\left(\operatorname{supp} \chi_{j}, A\right), \quad j=1,2, \cdots$

for some constant $C$.

In the following we quote each of (i), (ii), (iii) as the property of $\chi_{\jmath}$. Let $x \in \operatorname{supp} \chi_{\text {, }}$. Then it can be easily verified $d(x, A)>1$, provided $s_{\jmath}=1$ (see [4]). So we note $d(x, A) \leqq 1$ implies $s_{\jmath}<1$.

Proof of Lemma. Since $F$ is regular, to each $x \in F$ there corresponds an open ball $B_{d}(x)$ of center $x$, with radius $d$ in $\Omega$ such that any two points $y, z$ of $F \cap \overline{B_{d}(x)}$ can be joined by a rectifiable curve in $F$. Here we note the radius $d$ depends on $x$. Since $F$ is compact, we can choose a finite family $\left\{B_{d_{1}}\left(x_{1}\right)\right.$, $\left.\cdots, B_{d_{m}}\left(x_{m}\right)\right\}$ from the open cover $\left\{B_{d}(x) \mid x \in F\right\}$ of $F$ so that

$$
F \subset B_{d_{1}}\left(x_{1}\right) \cup \cdots \cup B_{d_{m}}\left(x_{m}\right) .
$$

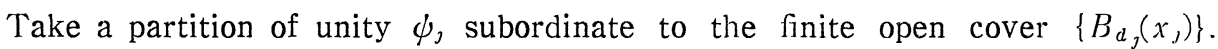
Then $u$ is represented in the form

$$
u=\dot{\varphi}_{1} u+\cdots+\phi_{m} u=u_{1}+\cdots+u_{m}
$$

where supp $u_{\jmath}=\operatorname{supp} \phi_{\jmath} u \subset F_{\jmath}=F \cap \overline{B_{d_{j}}\left(x_{\jmath}\right)}, F_{\jmath}$ being also compact and regular. Suppose the estimate (1) is valid for each $u_{j}, F_{j}, q$, instead of $u, F, q$. Then for $\varphi \in C^{\infty}(\Omega)$, there is a constant $C_{j}$ such that

$$
\left|\left\langle u_{\jmath}, \varphi\right\rangle\right| \leqq C_{\jmath}\|\varphi\|_{m_{j}, F_{j}} .
$$

where $m_{j}$ is any integer $\geqq m q_{\jmath}, q_{\text {, }}$ being a number $(\geqq 1)$ related to the regularity of $F_{\jmath}$. Clearly, Lemma is a consequence of (2) and (3), with $q=q(F)=\sup _{1 \leqq j \leq m} q_{\jmath}$ and $C=\sup _{1 \leqq \jmath \leqq m} C_{\jmath}$. So it suffices to derive (3) for each $\varphi \in C^{\infty}(\Omega)$, in which we write $F=F_{\jmath}$ and $q=q_{\jmath}$, dropping the subscript $j$ for simplicity of notation.

Take $\varphi$ in $C_{0}^{\infty}(\Omega)$ and extend it to a function in $C_{0}^{\infty}\left(R^{n}\right)$ by setting zero outside $\Omega$, which we denote $\varphi$ again. We shall give a function $\phi \in C^{m}(\Omega)$ so that $\phi^{(a)}(x)=\varphi^{(a)}(x)$ in $F$ when $|\alpha| \leqq m$ where $D^{a} f=f^{(a)}$. This can be carried out by the method of Whitney's extension theorem, as follows. Making use of the partition of unity $\chi_{j}$ in $R^{n} \backslash F$ just constructed above, we define a function $\psi$ by

$$
\psi(x)=\left\{\begin{array}{lll}
\varphi(x) & \text { for } \quad x \in F \\
\Sigma_{j}^{\prime} \chi_{\jmath}(x) \varphi_{m}\left(x ; y^{j}\right) & \text { for } \quad x \in R^{n} \backslash F
\end{array}\right.
$$

where $y^{j} \in F$ is taken so that

$$
\begin{gathered}
d\left(\operatorname{supp} \chi_{\jmath}, F\right)=d\left(\operatorname{supp} \chi_{\jmath}, y^{j}\right) \\
\varphi_{m}(x ; y)=\sum_{|\alpha| \leqq m} \frac{1}{\alpha !} \varphi^{(a)}(y)(x-y)^{a}
\end{gathered}
$$

and $\Sigma_{j}^{\prime}$ stands for the sum with $s_{\jmath}<1$. Then $\phi \in C_{0}^{m}\left(R^{n}\right)$ and satisfies 


$$
D^{\alpha} \psi=D^{a} \varphi \quad \text { in } \quad F
$$

when $|\alpha| \leqq m([6])$. What we are going to obtain is the estimate

$$
\|\varphi\|_{m, \Omega} \leqq C\|\varphi\|_{m^{\prime}, F}
$$

where $C$ is a constant depending only on $m, q$ and $F$. Let $x \in \Omega \backslash F$ be fixed. Then $d(x, F)>0$. To derive (5) we divide the case into 1) $d(x, F)>1$ and 2) $d(x, F) \leqq 1$. It is enough to show for the case where $m \geqq 1$.

1) $d(x, F)>1$.

Differentiation of $\phi$ in (4) gives, by Leibniz's formula,

$$
\psi^{(\alpha)}(x)=\sum_{\beta+\gamma=\alpha} \sum_{j} \chi_{j}^{(\beta)}(x) \varphi_{m}^{(\gamma)}\left(x ; y^{j}\right) .
$$

Since $\Sigma_{j}^{\prime}\left|\chi_{j}^{(\beta)}(x)\right| \leqq 2 C_{\beta}$ by the property (ii) of $\left\{\chi_{\jmath}\right\}$, we have

$$
\left|\psi^{(a)}(x)\right| \leqq C^{\prime}{ }_{a}\|\varphi\|_{m, F}
$$

for a constant $C^{\prime}{ }_{a}$ when $|\alpha| \leqq m$, which implies (5).

2) $0<d(x, F) \leqq 1$.

In this case, as we noted before, $s_{j}<1$ provided $x \in \operatorname{supp} \chi_{\jmath}$. Hence we have $\Sigma_{j}^{\prime} \chi_{j}(x)=\Sigma_{j} \chi_{j}(x)=1$, which gives

$$
\psi(x)=\varphi_{m}(x ; y)+\sum_{j} \chi_{\jmath}(x)\left[\varphi_{m}\left(x ; y^{\jmath}\right)-\varphi_{m}(x ; y)\right]
$$

where $y \in F$ is so chosen as $d(x, F)=|x-y|$. Further we take $x^{j} \in \operatorname{supp} \chi_{j}$ so as to satisfy $d\left(\operatorname{supp} \chi_{j}, F\right)=\left|x^{\jmath}-y^{\jmath}\right|$. Then

$$
\left|x-x^{j}\right| \leqq \operatorname{diam}\left(\operatorname{supp} \chi_{j}\right) \leqq C d\left(\operatorname{supp} \chi_{j}, F\right) \leqq C d(x, F)
$$

for some constant $C$ where we used the property (iii) of $\left\{\chi_{j}\right\}$. Thus in view of the definitions of $y, x^{j}$ and $y^{j}$, we get the inequalities

$$
\begin{aligned}
& \left|x-y^{\jmath}\right| \leqq\left|x-x^{\jmath}\right|+\left|x^{j}-y^{\jmath}\right| \leqq(C+1) d(x, F) \\
& \left|y-y^{\jmath}\right| \leqq|y-x|+\left|x-y^{\jmath}\right| \leqq(C+2) d(x, F)
\end{aligned}
$$

which will be needed later. Denoting by $R_{m}(x ; y)$ the remainder term of Taylor's formula at $y$, we have

$$
\varphi(x)=\varphi_{m}(x ; y)+R_{m}(x ; y) .
$$

Our basic concern is to estimate the derivatives of the difference $\varphi_{m}\left(x ; y^{y}\right)-$ $\varphi_{m}(x ; y)$ in (6). The Taylor polynomial of $\varphi^{(r)}\left(z^{\prime \prime}\right)$

$$
\varphi_{m}^{(\gamma)}\left(z^{\prime \prime} ; z^{\prime}\right)=\sum_{|\beta| \leq m-|\gamma|} \frac{1}{\beta !} \varphi^{(\beta+\gamma)}\left(z^{\prime}\right)\left(z^{\prime \prime}-z^{\prime}\right)^{\beta}
$$

combined with the formula for any $z, z^{\prime}, z^{\prime \prime}$

$$
\varphi^{(\beta+\gamma)}\left(z^{\prime}\right)=\varphi_{m}^{(\beta+\gamma)}\left(z^{\prime} ; z\right)+R_{m}^{(\beta+\gamma)}\left(z^{\prime} ; z\right)
$$


obtained by differentiating (8), gives

$$
\varphi_{m}^{(\gamma)}\left(z^{\prime \prime} ; z^{\prime}\right)=\sum_{|\hat{o}| \leqq m-|\not i|} \frac{1}{\delta !}\left[\varphi_{m}^{(\gamma+\grave{o})}\left(z^{\prime} ; z\right)+R_{m}^{(\gamma+\delta)}\left(z^{\prime} ; z\right)\right]\left(z^{\prime \prime}-z^{\prime}\right)^{\delta}
$$

On the other hand

$$
\varphi_{m}^{(\gamma)}\left(z^{\prime \prime} ; z\right)=\sum_{|\delta| \leq m-|\gamma|} \frac{1}{\delta !} \varphi_{m}^{(\gamma+\delta)}\left(z^{\prime} ; z\right)\left(z^{\prime \prime}-z^{\prime}\right)^{\delta}
$$

Thus the subtraction of (10) from (9) yields

$$
\varphi_{m}^{(\gamma)}\left(z^{\prime \prime} ; z^{\prime}\right)-\varphi_{m}^{(\lambda)}\left(z^{\prime \prime} ; z\right)=\sum_{|\delta| \leq m-|\gamma|} \frac{1}{\delta !} R_{m}^{(\gamma+\eta)}\left(z^{\prime} ; z\right)\left(z^{\prime \prime}-z^{\prime}\right)^{\delta},
$$

so that changing $z, z^{\prime}, z^{\prime \prime}$ to $y, y^{3}, x$ gives

$$
\begin{aligned}
\left|\varphi_{m}^{(i)}\left(x ; y^{j}\right)-\varphi_{m}^{(\gamma)}(x ; y)\right| & \leqq \sum_{|\eta| \leqq m-|\eta|}\left|R_{m}^{(\gamma+\eta)}\left(y^{j} ; y\right)\left(\lambda-y^{j}\right)^{\eta}\right| \\
& \leqq C_{0}\|\varphi\|_{m^{\prime}, F}+\sum_{|\eta| \leqq m^{\prime}-\{\gamma \mid}\left|R_{m^{\prime}}^{(\gamma+\eta)}\left(y^{j} ; y\right)\left(x-y^{j}\right)^{\eta}\right|
\end{aligned}
$$

since $R_{m}(x ; y)=\varphi_{m^{\prime}}(x ; y)-\varphi_{m}(x ; y)+R_{m^{\prime}}(x ; y)$, where $m^{\prime}$ is any integer $\geqq m q$. So we are left with estimation of $R_{m^{\prime}}^{(?+\eta)}\left(y^{j} ; y\right)$. This will be worked out by a technical modification of [7]. As $y, y^{\jmath} \in F$, there is a rectifiable curve $\mathcal{C}$ in $F$ of length, say $L$, joining $y$ and $y^{3}$. Let $\Delta: y=z^{0}, z^{1}, \cdots, z^{p}=y^{j}$ be a subdivision of $\mathcal{C}$ in $F$ and let $|\Delta|=\sup _{1 \leqq i \leqq p}\left|z^{i}-z^{2-1}\right|$. Note that

$$
\varphi_{m^{\prime}}^{(j)}\left(z^{\prime \prime} ; z^{\prime}\right)-\varphi_{m^{\prime}}^{(i)}\left(z^{\prime \prime} ; z\right)=R_{m^{\prime}}^{(\gamma)}\left(z^{\prime \prime} ; z\right)-R_{m^{\prime}}^{(r)}\left(z^{\prime \prime} ; z^{\prime}\right)
$$

since

$$
\begin{aligned}
\varphi^{(i)}\left(z^{\prime \prime}\right) & =\varphi_{m^{\prime}}^{(i)}\left(z^{\prime \prime} ; z\right)+R_{m^{\prime}}^{(\gamma)}\left(z^{\prime \prime} ; z\right) \\
& =\varphi_{m^{\prime}}^{(\gamma)}\left(z^{\prime \prime} ; z^{\prime}\right)+R_{m^{\prime}}^{(\gamma)}\left(z^{\prime \prime} ; z^{\prime}\right)
\end{aligned}
$$

Thus we get by (11)

$$
R_{m^{\prime}}^{(\gamma+\eta)}\left(z^{\prime \prime} ; z\right)-R_{m^{\prime}}^{(\eta+\eta)}\left(z^{\prime \prime} ; z^{\prime}\right)=\sum_{|\hat{o}| \leqq m^{\prime}-|\gamma+\eta|} \frac{1}{\delta !} R_{m}^{(\gamma+\eta+\delta)}\left(z^{\prime} ; z\right)\left(z^{\prime \prime}-z^{\prime}\right)^{\grave{\delta}} .
$$

Changing $z, z^{\prime}, z^{\prime \prime}$ to $z^{2-1}, z^{2}, y^{\jmath}$ in this equation, summing over $i$ and noting $R_{m^{\prime}}^{(\kappa)}\left(y^{j} ; y^{j}\right)=0$ when $|\kappa| \leqq m^{\prime}$, we consequently have

$$
R_{m}^{(\gamma+\eta)}\left(y^{\jmath} ; y\right)=\sum_{i=1}^{p} \sum_{\left|\delta_{1} \leqq m^{\prime}-\right| \gamma+\eta \mid} \frac{1}{\delta !} R_{m}^{\left(\gamma+\hat{o}+\gamma^{\prime}\right)}\left(z^{i} ; z^{i-1}\right)\left(y^{\jmath}-z^{2}\right)^{\grave{\delta}} .
$$

Note that by the classical formula for the remainder term

$$
\left|R_{m^{\prime}}^{(\gamma+\delta+\eta)}\left(z^{i} ; z^{i-1}\right)\right| \leqq\left|z^{i}-z^{i-1}\right|^{m^{\prime}-|\gamma+\delta+\eta|} \varepsilon\left(\left|z^{i}-z^{2-1}\right|\right)
$$

where $\varepsilon(h) \rightarrow 0$ when $h \rightarrow 0$.

Now split the sum for $\delta$ in (13) into the sums for $|\delta|<m^{\prime}-|\gamma+\eta|$ and for $|\delta|=m^{\prime}-|\gamma+\eta|$, and then denote the former by $I_{\lrcorner}$and the latter by $J_{\Delta}$, res- 
pectively. Since $\left|z^{2}-z^{i-1}\right| \leqq L$ and $\left|y^{\jmath}-z^{2}\right| \leqq L$, in view of (14) we have

$$
\begin{aligned}
\left|I_{\Delta}\right| & \leqq \sum_{|\delta|<m^{\prime}-|\gamma+\eta|} \frac{1}{\delta !} L^{m^{\prime}-|\gamma+\eta|} \sum_{i=1}^{p}\left|z^{i}-z^{i-1}\right| \varepsilon(|\Delta|) \\
& \leqq \sum_{|\delta|<m^{\prime}-|\gamma+\eta|} \frac{1}{\delta !} L^{m^{\prime}+1-|\gamma+\eta|} \varepsilon(|\Delta|)
\end{aligned}
$$

which tends to 0 when $|\Delta| \rightarrow 0$.

On the other hand

$$
J_{\Delta}=\sum_{i=1}^{p} \sum_{|\delta|=m^{\prime}-|\gamma+\eta|} \frac{1}{\delta !}\left[\varphi_{m}^{(\gamma+\varepsilon+\eta)}\left(z^{2}\right)-\varphi_{m}^{(\gamma+\delta+\eta)}\left(z^{i-1}\right)\right]\left(y^{\jmath}-z^{2}\right)^{\delta}
$$

since for $|\boldsymbol{\delta}|=m^{\prime}-|\gamma+\eta|$,

and so

$$
\varphi_{m^{\prime}}^{(\gamma+\tilde{o}+\eta)}\left(z^{2} ; z^{i-1}\right)=\varphi^{(\gamma+\delta+\eta)}\left(z^{\imath-1}\right)
$$

$$
\varphi^{(\gamma+\delta+\eta)}\left(z^{i}\right)=\varphi^{(\gamma+\delta+\eta)}\left(z^{i-1}\right)+R_{m^{\prime}}^{(\gamma+\delta+\eta)}\left(z^{2} ; z^{2-1}\right) .
$$

Now for each fixed $\delta$ we have

$$
\begin{gathered}
\sum_{i=1}^{p}\left[\varphi^{(\gamma+\delta+\eta)}\left(z^{i}\right)-\varphi^{(\gamma+\hat{\delta}+\eta)}\left(z^{i-1}\right)\right]\left(y^{j}-z^{i}\right)^{\hat{\delta}} \\
=-\sum_{i=1}^{p-1}\left[\varphi^{(\gamma+\delta+\eta)}\left(z^{i}\right)-\varphi^{(\gamma+\delta+\eta)}\left(z^{0}\right)\right]\left[\left(y^{j}-z^{i+1}\right)^{\grave{\delta}}-\left(y^{j}-z^{i}\right)^{\grave{\delta}}\right],
\end{gathered}
$$

which tends to a Stieltjes integral

$$
-\int_{0}^{L}\left[\varphi^{(\gamma+\hat{o}+\eta)}(z(s))-\varphi^{(\gamma+\hat{o}+\eta)}\left(z^{0}\right)\right] d\left(y^{j}-z(s)\right)^{\delta}
$$

when $|\Delta| \rightarrow 0$, where $z(s)$ denotes the point on the curve $\mathcal{C}$ of length $s$ along $C$ from $y$.

After the differentiation in the integral, (15) becomes

$$
\sum_{|\kappa|=1} \frac{\delta !}{(\delta-\kappa) !} \int_{y}^{y^{j}}\left[\varphi^{(\gamma+\hat{o}+\eta)}(z)-\varphi^{(\gamma+\hat{o}+\eta)}(y)\right]\left(y^{j}-z\right)^{\hat{\delta}-\kappa}(d z)^{\kappa} .
$$

Denote the sum of integrals (16) by $I_{i, \tilde{o}, \eta}$. Then we have

$$
R_{m}^{(\gamma+\eta)}\left(y^{j} ; y\right)=\sum_{|\tilde{o}|=m^{\prime}-|\gamma+\eta|} \frac{1}{\delta !} I_{\gamma, \delta, \eta}=\lim _{|\Delta| \rightarrow 0} J_{\Delta}
$$

Hence, taking the regularity of $F$ into consideration, we have the estimates

$$
\begin{aligned}
\left|R_{m^{\prime}}^{(\gamma+\eta)}\left(y^{j} ; y\right)\right| & \leqq C_{1} L^{m^{\prime}-|\gamma+\eta|}\|\varphi\|_{m^{\prime}, F} \\
& \leqq C_{1} d(x, F)^{\left(m^{\prime}-|\gamma+\eta|\right) / q}\|\varphi\|_{m^{\prime}, F}
\end{aligned}
$$

for some constant $C_{1}$ and for any $\gamma$ and $\eta$ with $|\gamma+\eta| \leqq m^{\prime}$. The last estimate combined with (7) and (12) implies 


$$
\left|\varphi_{m^{\prime}}^{(\gamma)}\left(x ; y^{\jmath}\right)-\varphi_{m}^{(\gamma)}(x ; y)\right| \leqq C_{2}\|\varphi\|_{m^{\prime}, F^{\prime}} \sum_{|\eta| \leqq m^{\prime}-|\gamma|} d(x, F)^{\left(m^{\prime}-|\gamma+\eta|\right) / q} d(x, F)^{|\eta|}
$$

where $C_{2}$ is a constant depending only on $m^{\prime}$ and $F$. Now, the differentiation of $\psi$ in (6) with respect to $x$ gives

$$
\psi^{(a)}(x)=\varphi_{m}^{(\alpha)}(x ; y)+\sum_{\beta+\gamma=\alpha} \sum_{j=1}^{\infty} \chi_{j}^{(\beta)}(x)\left[\varphi_{m}^{(\gamma)}\left(x ; y^{j}\right)-\varphi_{m}^{(j)}(x ; y)\right] .
$$

Thus in view of the property (ii) of $\left\{\chi_{j}\right\}$,

$$
\begin{aligned}
\left|\psi^{(\alpha)}(x)\right| \leqq & \|\varphi\|_{m, F} \\
& +C_{2}\|\varphi\|_{m^{\prime}} F_{\beta+\gamma=a}\left(d(x, F)^{-|\beta|}+1\right) \sum_{|\eta| \leqq m^{\prime}-|\gamma|} d(x, F)^{\left(\left(m^{\prime}-|\gamma+\eta|\right) / q\right)+|\eta|} .
\end{aligned}
$$

As for the exponent of $d(x, F)$, if $|\alpha| \leqq m$

$$
\begin{aligned}
\left(\left(m^{\prime}-|\gamma+\eta|\right) / q\right)+|\eta|-|\beta| & \geqq \frac{1}{q}\{m q-|\alpha-\beta|+(q-1)|\eta|-q|\beta|\} \\
& =\frac{1}{q}\{q(m-|\beta|+|\eta|)-(|\alpha|-|\beta|+|\eta|)\} \\
& \geqq \frac{q-1}{q}(m-|\beta|+|\eta|) \geqq 0 .
\end{aligned}
$$

Since $d(x, F) \leqq 1$, we finally have

$$
\left|\psi^{(\alpha)}(x)\right| \leqq C\|\varphi\|_{m^{\prime}, F}
$$

when $|\alpha| \leqq m$, where $C$ is a constant depending only on $m^{\prime}$ and $F$. Collecting the results obtained so far, we consequently proved the estimate (5). Recall a property of distributions with compact support that if $\chi \in C_{0}^{\infty}(\Omega)$ and its derivatives of order up to $m$ vanish on $F$, then $\langle u, \chi\rangle=0$ (cf. [6]). Suppose $\eta \in C_{0}^{\infty}(\Omega)$ is equal to 1 on a neighborhood of $F$. Then $\eta \psi$ can be regarded as a function in $C_{0}^{\infty}(\Omega)$ and $(\eta \varphi)^{(\alpha)}=(\eta \psi)^{(a)}$ on $F$ when $|\alpha| \leqq m$. Thus we get

and so

$$
\langle u, \eta \varphi\rangle=\langle u, \eta \psi\rangle \text {, }
$$

$$
\begin{aligned}
|\langle u, \varphi\rangle| & =|\langle u, \eta \varphi\rangle|=|\langle u, \eta \psi\rangle| \\
& \leqq C_{1}\|\phi\|_{m \Omega} \\
& \leqq C_{2}\|\varphi\|_{m^{\prime} F} \quad \text { (by (5)) }
\end{aligned}
$$

for any integer $m^{\prime} \geqq m q$ where $C_{1}, C_{2}$ are constants depending only on $m^{\prime}$ and $F$ which completes the proof of Lemma.

Remark. A typical example of regular set is a convex set, where $q=1$. In this particular case, the proof of Lemma is carried out much more readily than the above, since it is enough to use the classical formula for $R_{m}^{(i+\eta)}\left(y^{j} ; y\right)$ in (12). Today, we know a large family of regular sets, that is, compact sub- 
analytic sets in $R^{n}$ (or in real analytic manifolds) (see [1], [3]).

\section{Acknowledgement}

The author thanks the referee for his advice.

\section{References}

[1] Bierstone, E., Differentiable functions, Bol. Soc. Brasil Mat., II (1980), 139-180.

[2] Glaeser, G., Etude de quelques algèbres tayloriennes, J. Anal. Math., 6 (1958), 1124.

[3] Hardt, R.M., Some analytic bounds for subanalytic sets, Differential geometric control theory, Birkhäuser, Boston (1983), 259-267.

[4] Hörmander, L., On the division of distributions by polynomials, Ark. Mat., 3 (1958), $555-568$.

[5] Mizohata, S., On evolution equations with finite propagation speed, Israel Jour. of Math., 13 (1972), 173-185.

[6] Schwartz, L., Théorie des distributions, Hermann, Paris, 1966.

[7] Whitney, H., Functions differentiable on the boundaries of regions, Ann. of Math., 35 (1934), 482-485.

[8] - Analytic extensions of differentiable functions difined in closed sets, Trans. Amer. Math. Soc., 36 (1934), 63-89.

[9] - On the extension of differentiable functions, Bull. AMS, 50-2 (1944), $76-81$. 